\title{
El pasado pisado. Investigaciones arqueológicas en tecnología y tafonomía líticas de los cazadores recolectores de sierra Baguales, SO de Santa Cruz, Argentina
}

\section{(7) Catalina Balirán ${ }^{*}$}

Fecha de defensa: 21 de septiembre de 2020

Directora: Dra. Karen Borrazzo

Co-director: Dr. Luis A. Borrero

Jurado: Dres. Nora V. Franco y Rafael A. Goñi

El objetivo de esta tesis fue contribuir al conocimiento arqueológico de la localidad arqueológica La Verdadera Argentina (LVA), ubicada al sudeste de sierra Baguales (Santa Cruz, Argentina) y su rol dentro del rango de acción de las poblaciones que ocuparon el extremo sudoeste de Patagonia continental. Este objetivo se llevó a cabo a través del estudio tecno-morfológico y tafonómico del conjunto de materiales líticos provenientes de la recolección superficial denominada Transecta 9 (T9).

Emplazada entre 300 y 600 m.s.n.m., LVA cuenta con precipitaciones de $300 \mathrm{~mm}$ anuales, una temperatura media de $8{ }^{\circ} \mathrm{C}$ y vientos de hasta $120 \mathrm{~km} / \mathrm{h}$. La vegetación es de tipo estepa arbustiva baja (Oyarzabal et al., 2018). En el área habitan especies animales nativas y domesticadas. En cuanto a las fuentes de materias primas, las mismas son principalmente de tipo secundario e incluyen varias litologías disponibles bajo la forma de nódulos y/o grandes bloques como las denominadas lutitas o rocas lutíticas (entre las que se identificaron con cortes petrográficos fangolitas, lutitas y grauvacas, Borrazzo, 2008), ftanita, dacita y diabasa. Asimismo, se identificaron fuentes primarias (Borrazzo, 2008) y secundarias (Franco y Aragón, 2004) de calcedonia, que se suman a la ya conocida obsidiana gris verdosa veteada con procedencia de sierra Baguales (Borrero et al., 2006; Stern y Franco, 2000).

Respecto a las ocupaciones humanas, en LVA se obtuvieron fechados que abarcan todo el Holoceno ( $8856 \pm 84$ al $907 \pm 45$ años 14C AP, Borrero y Borrazzo, 2011). Los estudios llevados a cabo en la localidad mostraron que el registro arqueológico superficial es el más frecuente. Allí se utilizaron materias primas locales pero son las rocas lutíticas las que dominan los conjuntos. Estas fueron utilizadas principalmente en el marco de estrategias expeditivas. El uso del espacio habría sido de tipo logístico, con un predominio de estrategias de equipamiento del espacio durante el Holoceno temprano y de equipamiento de los individuos durante el Holoceno tardío (Borrazzo, 2008; Kuhn, 2004). Finalmente los estudios arqueofaunísticos evidenciaron

* Instituto Multidisciplinario de Historia y Ciencias Humanas (IMHICIHU), CONICET. Saavedra 15, $5^{\circ}$ piso (CP C1083ACA) Ciudad Autónoma de Buenos Aires, Argentina. E-mail: cataclorofila@gmail.com que la localidad habría sido utilizada principalmente en los meses de primavera y verano, lo cual es consistente con las condiciones de estrés invernal planteadas para el área (L’Heureux y Borrazzo, 2013; Pallo, 2012). En función de la evidencia mencionada, LVA fue definida como un área marginal dentro de los rangos de acción de las poblaciones localizadas al Este de este espacio (Borrero et al., 2006; Franco et al., 1999; L'Heureux y Borrazzo, 2013). Acorde a esto, en esta tesis se planteó como hipótesis tecnológica que en T9 hubo un predominio de estrategias tecnológicas de tipo expeditivas y/u oportunistas similares a las encontradas en otros sitios de la localidad.

En cuanto a los antecedentes tafonómicos en LVA, desde su inicio, los trabajos llevados a cabo allí por nuestro equipo se insertaron dentro de un marco de trabajo que incluyó este tipo de estudios, esto permitió contar con información acerca de diferentes procesos y agentes actuando en la localidad y sus posibles efectos sobre los conjuntos líticos (Borrazzo, 2008, 2011; Borrero et al., 2006). Entre ellos se observó que los artefactos de lutita encontrados en superficie presentaban de forma recurrente parte de su superficie con estadios de meteorización menores al resto de la pieza. Estas superficies más frescas coincidían sistemáticamente con superficies de lascado que en algunos casos formaban filos.

En esta tesis se planteó que para el conjunto T9, el origen de estos filos podía ser producto tanto de la acción antrópica, mediante un proceso de reclamación sensu Schiffer (1987) o bien ser producto de la acción de procesos postdepositacionales, en particular del pisoteo de animales. Esta última opción se planteó como la hipótesis tafonómica.

Teniendo en cuenta estos antecedentes, y con el fin de cumplir con el objetivo general arriba mencionado, se plantearon dos objetivos particulares:

1. Estudiar y comprender el registro lítico de superficie de LVA a partir del análisis del material arqueológico obtenido en T9.

2. Realizar el estudio tafonómico de la muestra lítica de T9 y conducir experimentos con seguimiento longitudinal que evalúen la incidencia del pisoteo de animales sobre el registro lítico de superficie.

Para cumplir con los objetivos propuestos se realizaron análisis tafonómicos y tecnomorfológicos de la muestra de T9 siguiendo la propuesta de Borrazzo (2004) y Aschero 
$(1975,1983)$ respectivamente. Asimismo se desarrolló un plan experimental in situ cuyo diseño apuntó a fortalecer la validez interna de los resultados (Borrero, 1991; Lin, Rezek y Dibble, 2018) y que permitió obtener información acerca de la acción de agentes y procesos postdepositacionales en LVA.

El análisis tafonómico de T9 $(\mathrm{N}=329)$ permitió el reconocimiento de un número considerable de pseudoartefactos $(\mathrm{N}=22)$, los cuales debieron ser excluidos de la muestra para los siguientes análisis. De esta forma la muestra de T9 sujeta a los estudios tecnomorfológicos fue de 307 artefactos. Para el estudio de la meteorización de la lutita se desarrollo una escala siguiendo la propuesta de Hiscock (1985) donde se definieron cuatro estadios de 0 a 3, donde 0 es sin meteorización y 3 la máxima meteorización observada. De los 307 artefactos de T9, 152 presentaron distintos estadios de alteración sobre la superficie de al menos una de sus caras, en 73 casos el estadio menos intenso coincidió con el filo de las piezas y se trató en todos los casos de los estadios de meteorización 0 $(\mathrm{N}=72)$ y $1(\mathrm{~N}=1)$ mientras que el estadio máximo identificado en dichos artefactos varió entre 1 y 3 . En los 73 casos, se trató de artefactos de lutita. Si se considera al tiempo como una variable clave en el desarrollo de la capa meteorizada de los artefactos (Colman, 1977; Glauberman y Thorson, 2012), podemos asumir que mientras los artefactos fueron probablemente confeccionados en diferentes momentos de la historia de LVA, el daño observado en los filos se habría producido en algún momento más reciente. Asimismo, la meteorización similar registrada en estos filos sugiere una relativa penecontemporaneidad. Estas características podrían ser explicadas como producto del pisoteo animal, sobre todo si se considera la introducción de ganado europeo (vacas, ovejas y caballos) en los últimos 120 años (Mancini, 2002; Martinic, 2000).

Los resultados experimentales mostraron que el pisoteo animal era el único proceso presente en LVA con el potencial de generar daño en los artefactos (fracturas y pseudolascados). Sin embargo los cambios observados en las pistas experimentales dieron cuenta de la existencia de otros factores que contribuyen a la dinámica a la que se encuentra expuesto el registro superficial en la localidad, tales como procesos asociados a la pendiente, la acción de aguas de escorrentía y crioturbación (Ozán, 2017; Waters, 1992).

Si bien los datos experimentales no son suficientes como para descartar la acción antrópica en la generación de los filos observados, introducen al pisoteo animal como una opción parsimoniosa a ser considerada al momento de analizar este tipo de características en los artefactos de superficie de LVA. La comparación entre los datos obtenidos en el análisis tafonómico de T9 y los datos experimentales permitieron reconocer características comunes entre ambos conjuntos. Los 73 filos observados en los artefactos de lutita de T9 mostraron diferencias sustanciales respecto de los instrumentos formales presentes en dicho conjunto, por lo que los filos con menor estadio de meteorización no fueron tenidos en cuenta en los análisis tecnológicos.

El análisis tecnológico del conjunto T9 mostró una tendencia predominante hacia la priorización de estrategias expeditivas. Las características tecnomorfológicas de los núcleos de lutita de T9 fueron consistentes con un escenario de cercanía a las fuentes y/o abundancia de materias primas (Byrne, 1980; Odell, 2000; Shott y Nelson, 2008). Esto guardaba coherencia con la presencia de una cantera taller en las cercanías de T9. Esta información fue consistente con la aportada por otros conjuntos de LVA (Borrazzo, 2008; Borrero et al., 2006).

Por otro lado en T9 se identificó la presencia de dos artefactos confeccionados sobre roca de grano fino oscuro tipo Potrok Aike (RFGO PKA, sensu Charlin y Pallo, 2013), materia prima no identificada localmente y cuya área fuente fue identificada en el Campo Volcánico Pali Aike (CVPA), ubicado a ca. 200 $\mathrm{km}$ al Sudeste de la localidad. Si bien el hallazgo en el CVPA de grandes instrumentos confeccionados sobre lutita, cuya proveniencia podría ser de LVA, llevaron a plantear la relación entre estas dos áreas (Borrazzo y Cirigliano, 2020; Pallo, Cirigliano, Charlin y Borrazo, 2020), hasta el momento no se había encontrado evidencia de dicha relación en LVA. A nivel general el registro de T9 es consistente con lo planteado a la fecha para la localidad. Sin embargo, algunos de los datos obtenidos sugieren que los sitios estudiados hasta el momento no serían representativos del total de las actividades llevadas allí a cabo. En este sentido LVA podría haber representado un área fuente privilegiada (por calidad, cantidad y tamaño) de lutita. Si esto fuera así, es posible que su importancia dentro de los circuitos de movilidad de las poblaciones que habitaron la región durante el Holoceno haya sido mayor que la hasta ahora propuesta (Borrero et al., 2006; Franco et al., 1999). Estos resultados plantean la necesidad de que los trabajos futuros en LVA aborden el estudio de los loci de aprovisionamiento de materias primas líticas, es decir, las canteras-taller.

\section{Referencias citadas}

"Aschero, C. (1975). Ensayo para una clasificación morfológica de artefactos líticos aplicada a estudios tipológicos comparativos. Apéndices AC. Revisión. Buenos Aires: Cátedra de Ergología y Tecnología, Facultad de Filosofía y Letras, Universidad de Buenos Aires. Manuscrito Inédito.

» Aschero, C. (1983). Ensayo para una Clasificación Morfológica de Artefactos Líticos Aplicada a Estudios Tipológicos Comparativos. Apéndices AC. Revisión. Buenos Aires: Cátedra de Ergología y Tecnología, Facultad de Filosofía y Letras, Universidad de Buenos Aires. Manuscrito Inédito.

» Borrazzo, K. (2004). Hacia una tafonomía lítica: el análisis tafonómico y tecnológico de los conjuntos artefactuales 
líticos de superficie provenientes de los loci San Genaro 3 y 4 (Bahía San Sebastián - Tierra del Fuego, Argentina). (Tesis de Licenciatura inédita). Universidad de Buenos Aires, Argentina.

» Borrazzo, K. (2008). Análisis tecnológico de distribuciones artefactuales en la periferia sudeste de la sierra Baguales (Santa Cruz, Argentina). Magallania (Punta Arenas), 36(1), 103-116.

» Borrazzo, K. (2011). Tafonomía lítica en la estepa patagónica: experimentación y registro arqueológico de superficie. En L. A. Borrero y K. Borrazzo (Eds.), Bosques, montañas y cazadores. Investigaciones arqueológicas en Patagonia Meridional (pp. 127-153). Buenos Aires: Instituto Multidisciplinario de Historia y Ciencias Humanas (IMHICIHU), CONICET.

» Borrazzo, K. y Cirigliano, N. (2020). La organización de la tecnología lítica en el interfluvio Gallegos-Chico (Santa Cruz, Argentina): una meso-transecta a los $070^{\circ}$ en el Campo Volcánico Pali Aike. Relaciones de La Sociedad Argentina de Antropología, XLV(1), 175-199.

» Borrero, L. A. (1991). Experimentos y escalas arqueológicas. Shincal, 3(1), 142-145.

» Borrero, L. A. y Borrazzo, K. (2011). La geografía cultural del sudoeste de Patagonia continental. En L. A. Borrero y K. Borrazzo (Eds.), Bosques, Montañas y Cazadores. Investigaciones Arqueológicas en Patagonia Meridional (pp. 7-36). Buenos Aires: Instituto Multidisciplinario de Historia y Ciencias Humanas (IMHICIHU), CONICET.

» Borrero, L. A., Franco, N. V., Martin, F. M., Barberena, R., Guichón, R., Belardi, J. B., Favier Dubois, C. y L'Heureux, G. L. (2006). Las cabeceras del Coyle: información arqueológica y circulación de poblaciones humanas. En J. B. Belardi, F. Carballo Marina, y S. L. Espinosa (Eds.), La Cuenca del Río Coyle. Estado Actual de las Investigaciones (pp. 75-95). Río Gallegos: Universidad Nacional de la Patagonia Austral.

" Byrne, D. (1980). Dynamics of dispersion: The place of silcrete in archaeological assemblages from the Lower Murchison, Western Australia. Archaeology and Physical Anthropology in Oceania, 15, 110-119.

"Charlin, J. E. y Pallo, M. C. (2013). Disponibilidad de materias primas líticas y uso del espacio en el interfluvio Gallegos-Chico (Pali Aike, Santa Cruz, Argentina). En A. Zangrando, R. Barberena, A. Gil, G. Neme, M. Giardina, L. Luna, C. Otaola, S. Paulides, L. Salgán y A. Tívoli (Comps.), Tendencias Teórico-Metodológicas y Casos de Estudio en la Arqueología de la Patagonia (pp. 307-316). Buenos Aires: Sociedad Argentina de Antropología.

" Colman, S. M. (1977). The Development of Weathering Rinds on Basalts and Andesites and their Use as a Quaternary Dating Method. (Tesis de Doctorado inedita), University of Colorado, Estados Unidos.

»Franco, N. V. y Aragón, E. (2004). Variabilidad en fuentes secundarias de aprovisionamiento lítico: el caso del sur del Lago Argentino. Estudios Atacameños, 28, 71-81.

"Franco, N. V., Borrero, L. A., Belardi, J. B., Carballo, F. M., Martin, F. M., Campán, P., Favier Dubois, C., Stadler, N., Hernández Llosas, M. I., Cepeda, H., Muñoz, A. S., Borella, F., Muñoz, F. y Cruz, I. (1999). Arqueología del cordón Baguales y sistema lacustre al sur del lago Argentino.
Praehistoria, 3, 65-86.

» Glauberman, P. J. y Thorson, R. M. (2012). Flint patina as an aspect of 'flaked stone taphonomy': A case study from the loess terrain of the Netherlands and Belgium. Journal of Taphonomy, 10(1), 21-43.

»Hiscock, P. (1985). The need for a taphonomic perspective in stone artefact analysis. Queensland Archaeological Research, 2, 82-97.

» Kuhn, S. L. (2004). Upper Paleolithic raw material economies at Üçağızlı Cave, Turkey. Journal of Anthropological Archaeology, 23(4), 431-448.

» L'Heureux, L. G. y Borrazzo, K. (2013). La arqueofauna del sudeste de Sierra Baguales durante el Holoceno: El sitio Cerro León 3 (Santa Cruz, Argentina). Magallania (Punta Arenas), 41(1), 223-240.

» Lin, S. C., Rezek, Z., y Dibble, H. L. (2018). Experimental design and experimental inference in stone artifact archaeology. Journal of Archaeological Method and Theory, 25(3), 663-688.

" Mancini, M. V. (2002). Vegetation and climate during the Holocene in Southwest Patagonia, Argentina. Review of Palaeobotany and Palynology, 122(3-4), 101-115.

» Martinic, M. (2000). Última Esperanza En El Tiempo. Punta Arenas: Ediciones de La Universidad de Magallanes.

» Odell, G. H. (2000). Stone tool research at the end of the millennium: Procurement and technology. Journal of Archaeological Research, 8(4), 269-331.

» Oyarzabal, M., Clavijo, J., Oakley, L., Biganzoli, F., Tognetti, P., Barberis, I., Maturo, H. M., Aragón, R., Campanello, P. y Prado, D. (2018). Unidades de vegetación de la Argentina. Ecología Austral, 28(1), 40-63.

» Ozán, I. L. (2017). Gravity and the formation of the archaeological record: Main concepts and methodological tools. Geoarchaeology, 32(6), 646-661.

" Pallo, M. C. (2012). El estrés invernal como generador de áreas marginales en el extremo sur de Patagonia Continental durante el Holoceno Tardío. Comechingonia Virtual: Revista Electrónica de Arqueología, 6(1), 86-114.

» Pallo, M. C., Cirigliano, N. A., Charlin, J. E. y Borrazzo, K. (2020). Una aproximación a la distribución de la producción laminar en la Patagonia Meridional. Revista del Museo de Antropología, 13(1), 217-222.

"Schiffer, M. B. (1987). Formation Processes of the Archaeological Record. Albuquerque: University of New Mexico Press.

»Shott, M. J. y Nelson, M. (2008). Lithic reduction, its measurement, and implications: Comments on the volume. En W. Andrefsky Jr. (Ed.), Lithic Technology: Measures of Production, Use, and Curation (pp. 23-46). Cambridge: Cambridge University Press.

»Stern, C. R. y Franco, N. V. (2000). Obsidiana gris verdosa veteada en la cuenca superior del río Santa Cruz, extremo sur de Patagonia. Anales del Instituto de la Patagonia, 28, 265-273.

» Waters, M. R. (1992). Principles of Geoarchaeology: A North American Perspective. Tucson: University of Arizona Press. 